\title{
Prevalence of Scaphoid Scapula in Indian Population and its Clinical Significance
}

\author{
Rajani Singh, ${ }^{1}$ N N Srivastava ${ }^{1}$ \\ 'Department of Anatomy UP University of Medical Sciences Saifai Etawah 206130, UP, India
}

Disclose and conflicts of interest: none to be declared by all authors

\begin{abstract}
Introduction: normal Scapulae possess three borders; superior, medial/vertebral and lateral. According to standard text books of Anatomy, Superior border is straight, vertebral border is convex and lateral border is straight. But during scanning of scapulae in the osteology lab in department of Anatomy UPUMS Saifai, scapulae with concave and straight vertebral border were detected. On literature search, it was found that such scapulae were designated scaphoid scapulae. Also, scanty literature describes such anomaly of scapulae. Hence the study was conducted. The aim of the study is to elucidate the prevalence of scaphoid scapulae in Indian Population and to bring out associated clinical significance.

Methods: : Vertebral border of fortyone scapulae, assorted and of unknown sex from osteology lab of UPUMS Saifai India, were observed to find the incidence of scaphoid scapulae.

Results: Out of total 41 scapulae observed, normal scapulae with convex vertebral border were observed in 19 (46.34\%) while scaphoid scapulae were detected in $22(53.66 \%)$. In category of scaphoid scapulae, scapulae with concave border were observed in $3(13.64 \%)$, with straight border in $12(54.55 \%)$ and those with mixed border in 7 (31.82\%) specimens.

Conclusion: literature states that this anomaly is congenital and associated with certain conditions such as nocturnal incontinence, catarrhal affections, adenoids, vasculosclerotic changes to name a few. If scaphoid scapulae is detected in developing fetus, one can predict associated anomalies and necessary precaution may be taken during rearing of child. Thus, knowledge of this anomaly is essential to clinicians, anthropologists and anatomists.
\end{abstract}

Keywords: Scapula; Scaphoid scapula; Race; Vertebral border.

\section{Introduction}

Scapula, also known as shoulder blade, is a paired triangular bone located on the posterior thoracic wall. It articulates with the head of humerus at glenohumeral joint and with the clavicle at the acromioclavicular joint. Scapula possesses three borders viz superior, medial and lateral. Superior border is characterised by suprascapular notch, lateral border extends from the infraglenoid tubercle to the inferior angle. Medial border also known as vertebral border, is normally convex and extends from superior angle to inferior angle. ${ }^{1}$ But sometimes vertebral border may appear concave or straight instead of convex. ${ }^{2}$ Such scapula with concave/straight vertebral border were named as scaphoid scapula. ${ }^{2}$ Scaphoid scapulae are more common in individuals who diverge from average physical and mental abilities. Persons of second generation whose parents contracted syphilis, scaphoid scapulae were found to be associated with nocturnal incontinence, catarrhal affections, adenoids, vasculosclerotic changes observed in very early in life. In aged persons, arteriosclerosis was found out of proportion of their age and this defect was detected even in individuals of third and fourth generations. ${ }^{2}$ There is paucity of literature elucidating the prevalence of scaphoid scapula in Indian Population. Hence the study was carried out. Aim of the study is to classify and describe the incidence of scaphoid scapula in Indian Population and to bring out associated clinical significance.

\section{Material and Methods}

Forty one assorted scapulae of unknown sex from UP University of Medical Sciences, Saifai UP India, were examined for the prevalence of scaphoid scapulae. Scapulae were designated as normal with convex medial border and scaphoid (concave, straight or with mixed bordered) Author of present study classify scapulae into three groups depending upon the appearance of vertebral/medial border of scapulae. Unossified, broken and damaged Scapulae were excluded from the study.

Type I: Normal scapulae with convex vertebral border (Fig. 1)

Type II: Scaphoid scapulae

Type II a: Scapula with concave vertebral border (Fig. 2)

Type IIb: Scapula with straight vertebral border (Fig. 3)

Type II c: Scapula with curved/mixed (combination of convex, concave or straight) vertebral border (Fig. 4)

Percentage of normal (Type I) and scaphoid scapulae (Type II.) were calculated and tabulated. Literature search was carried out and associated clinical significance was brought out. 

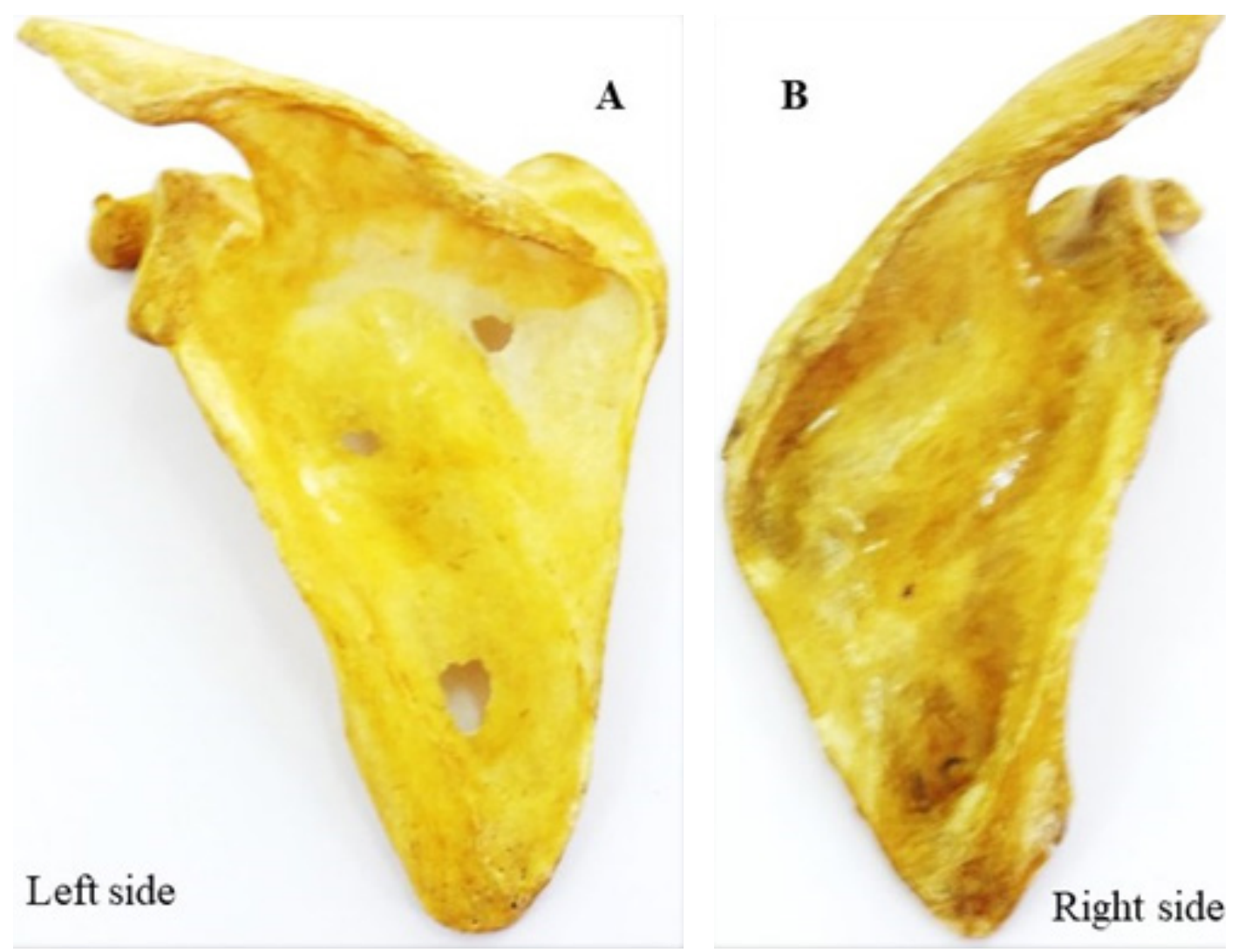

Figure 1. Normal scapulae with convex vertebral border
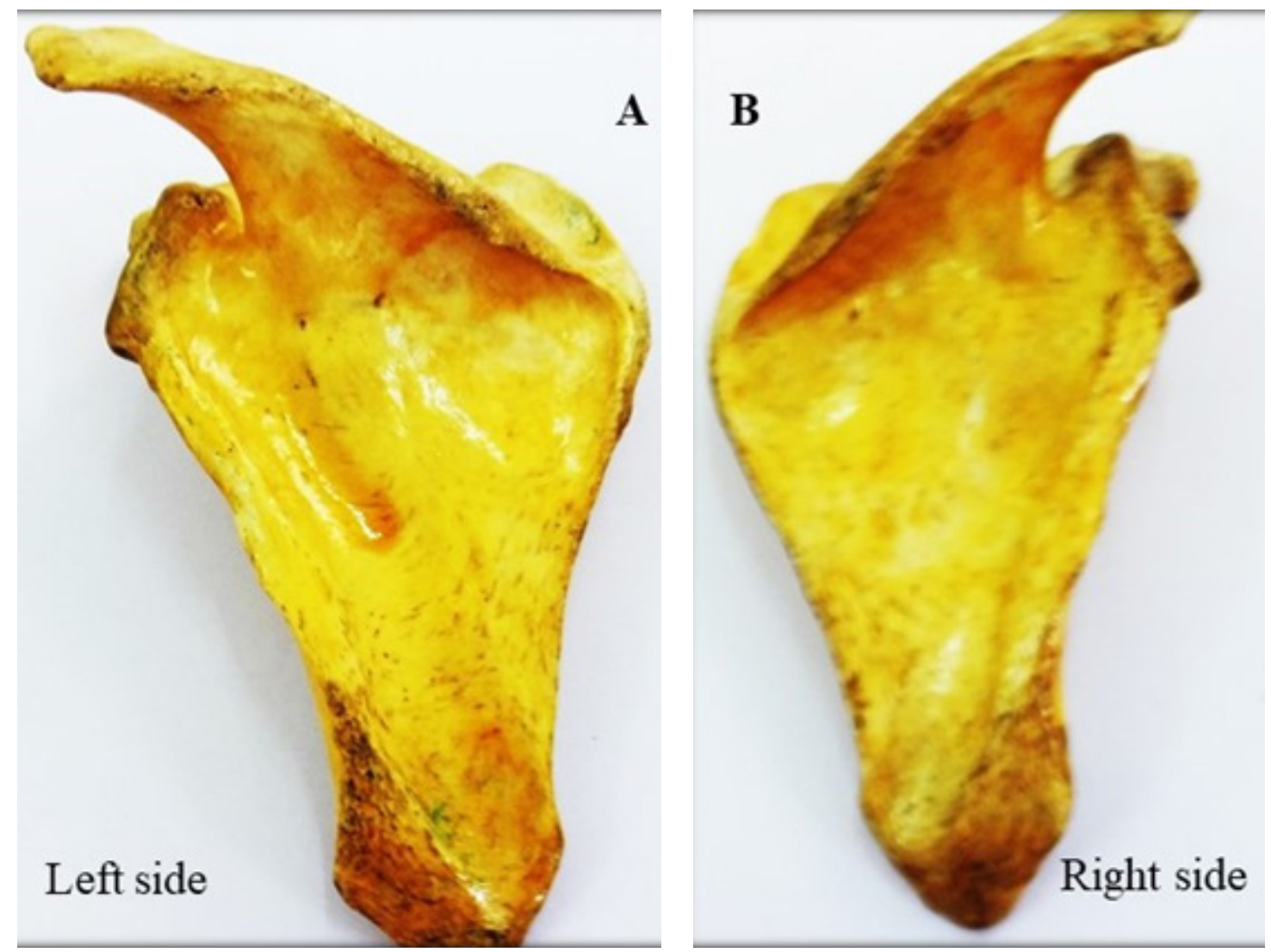

Figure 2. Scapulae with concave vertebral border 

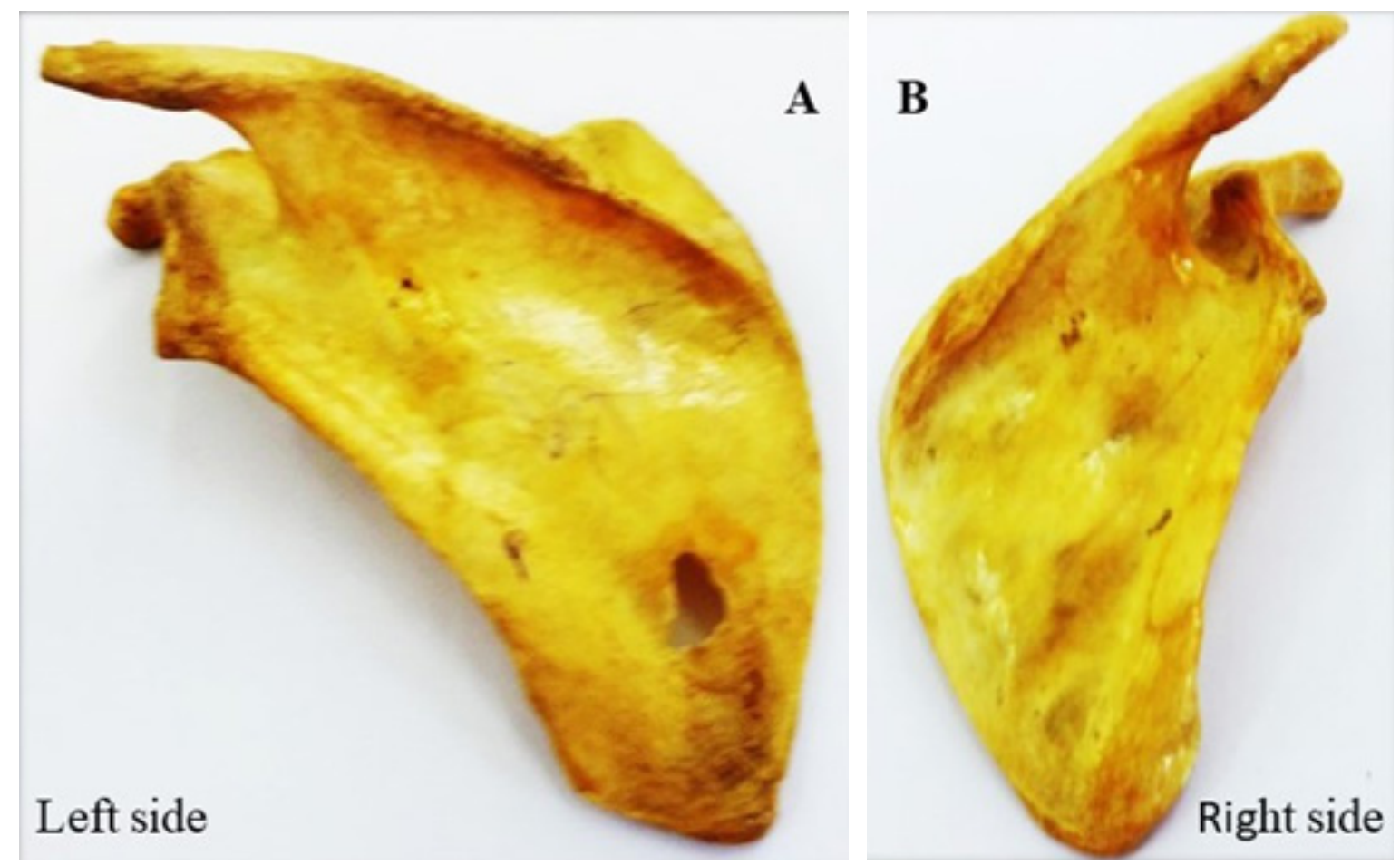

Figure 3. Scapulae with straight vertebral border

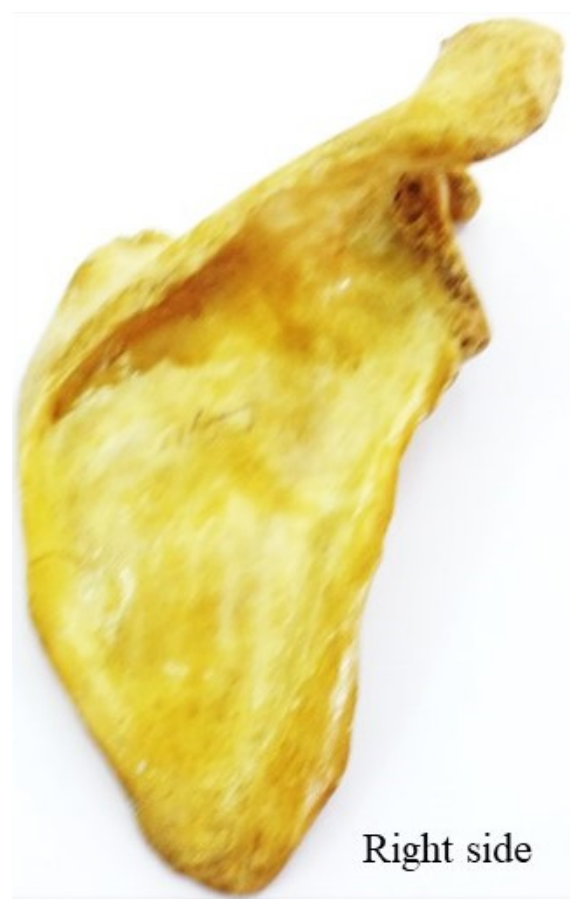

Figure 4. Scapula with mixed vertebral border

\section{Results}

Out of total 41 scapulae observed, normal scapulae with convex vertebral border were observed in 19 (46.34\%) while scaphoid scapulae were detected in 22 (53.66\%). In category of scaphoid scapulae, scapulae with concave border were observed in 3 (13.64\%), with straight border in 12 (54.55\%) and those with mixed border in 7 (31.82\%) specimens.

\section{Discussion}

Vertebral border of Scapulae from the medial end of scapular spine to inferior angle of the scapula could be convex, concave, straight or mixed. ${ }^{2}$ Graves classified scapulae as convex and scaphoid encompassing concave, straight and mixed in scaphoid scapulae. Few authors consider this anomaly as normal variant. ${ }^{3,4}$ The etiology of scaphoid scapulae is attributed to genetic and racial. Scaphoid scapula shows ethinicity related differences. ${ }^{2}$ In Indian Population, this anomaly was observed in approximately $54 \%$ of population. There is no data available to compare our results.

Scaphoid scapulae are more common in individuals who diverge from average physical and mental abilities. Persons of second generation whose parents suffered from syphilis, scaphoid scapulae were found to be associated with nocturnal incontinence, catarrhal affections, adenoids, vasculosclerotic changes observed in very early in life. In aged persons, arteriosclerosis was found out of proportion of their age and this defect was detected even in individuals of third and fourth generations. ${ }^{2}$ Quite high incidence of neuroses and psychoneuroses and especially epilepsy, dementia preacox and tuberculosis is found in such progeny. Patients of second and later generation with scaphoid scapula are more likely to have catarrhal affections, tonsillitis, bronchitis, gastro-intestinal disturbance and adenoids. ${ }^{2}$ Scaphoid scapulae may be genetic/congenital and racial and it is associated with multiple disorders. Hence if scapulae are diagnosed in fetus, various disorders associated with scaphoid scapula may be taken care of and treated.

In very thin individuals only scaphoid scapulae can 
be determined by inspection and palpation while in obese and muscular persons the $\mathrm{x}$-rav may supplement inspection and palpation. ${ }^{2}$

Appearance of persons with scaphoid scapula:

Outer appearance of individuals with scaphoid scapulae differ from those of normal individuals. In persons with scaphoid scapulae, chest is narrow in all directions, costosternal angle is narrow and have floating tenth rib. Such individuals have prominent acromioclavicular articulation. The shoulders drop downward and forward and vertebral borders are prominent. The hollowing out of the vertebral borders is well observed in thin persons with scaphoid scapulae.

Emil quoted that Prevalence of scaphoid more so concave bordered scapulae decreased after twenty years with corresponding increase of convex bordered and at the eighth decade, $80 \%$ of the scapulae were having convex vertebral border. ${ }^{5}$ In a group with some abnormality/unfitness like prisoners, people with feeble mind, concave type was more common than convex type. However, in another group consisting of soldiers, old people, honours students, convex type was more prevalent. But Graves did not find any evidence of change from one type of scapula to another type. ${ }^{5}$ According to Emil it could be due to personal error during physical examination. Change from slight convex to straight or from straight to slight concave may be missed during clinical examination. Personal error might have been affected by nutrition for a fat muscular border is more likely to be interpreted as convex irrespective of shape of border of scapula. ${ }^{5}$ $\mathrm{X}$-ray chest is more reliable method to designate scapula as convex bordered or scaphoid scapula.

\section{Authors view about the etiology of scaphoid scapula:}

The analysis of present study reveals that besides congenital, etiology of scaphoid scapula may be associated with environmental and occupational factors as it is observed in specific groups as elaborated in preceding sections. This fact can be elaborated as under- Serratus anterior muscle comprised of three part: Serratus anterior superior, Serratus anterior medial/intermediate and serratus anterior inferior. Serratus anterior superior is inserted into superior angle up to root of spine of scapula, Serratus anterior medial is inserted into medial border of scapula between root of the spine and inferior angle. Serratus anterior inferior is inserted into the inferior angle of the scapula. The rhomboideus major is inserted into dorsal surface of vertebral border of scapula between spine of scapula and inferior angle. The serratus anterior muscle protracts the scapula especially during pushing and punching movements and during overhead abduction of upper limb as during carrying heavy loads on head. Rhomboideus muscles retract the scapula. So, when serratus anterior muscle is excessively used in presence of weak rhomboideus major muscle or rhomboideus muscle is less used, scaphoid scapula especially with concave border, results. Thus, scaphoid scapula with concave border may also be caused by overuse of serratus anterior and weak rhomboideus muscle.

Straight bordered scapula may be produced when serratus anterior and rhomboideus major muscles are used equally or to the same extent. But further studies are to be carried out to confirm this fact.

Though it is claimed by few investigators that scaphoid scapula is hereditary caused by transmission of defective genes. ${ }^{2}$ But author of the study speculates based on the functions of muscles attached to the vertebral border that the condition may also be dependent on environmental/occupational factors in addition to genetic.

\section{Conclusion}

Individuals with Scaphoid scapulae suffer from multitude of abnormalities. Most of the studies state that it is congenital. So, if a child is found to be suffering from a disease characteristic of scaphoid scapulae, he should be screened for scaphoid scapula. If scaphoid scapula is detected other associated ailments should be looked for and treated. In Indian Population scaphoid scapula are observed in approximately $54 \%$ cases which is quite high. Hence in Indian Population, if scaphoid scapula is detected, the individual should be screened for associated disorders. In addition to this, scapulae with scaphoid border may help in designating race of the individual.

\section{References}

1. Standring S. (2005) Gray's Anatomy: The Anatomical basis of clinical practice. 40th Ed. Elsevier Churchill Livingstone. Spain, pp. 819

2. Graves WIlliam W. (1910) The Clinical Recognition Of The Scaphoid Type of Scapula and of some of its Correlations. JAMA. 55(1):12-17. doi:10.1001/jama.1910.04330010014006

3. Dwight T. (1887) The Range of Variation of the Human Shoulder-
Blade. The American Naturalist. 21: 627 - 638 DOI:10.1086/274521 4. Ruby L. Cunningham. The Scaphoid Scapula: A Normal Variation in Man Arch Intern Med (Chic). 1912; X (6):589-596. doi:10.1001/ archinte.1912.00060240071006

5. Emil Bogen, Arthur Rogers (1943) "The Scaphoid Scapula in Tuberculosis1." American Review of Tuberculosis, 47(3), pp. 303-307.
Received: April 19, 2021

Accepted: May 27, 2021
Corresponding author

Rajani Singh

E-mail: nani_sahayal@rediffmail.com 